\title{
Induction Immunosuppression in Pediatric Kidney Transplant Recipients Under the Age of 11 with Rabbit Anti-Thymocyte Globulin
}

\author{
William C. Goggins, MS1 ${ }^{1}$, Richard Mangus, MD ${ }^{1,2}$, Burcin Ekser, MD, $\mathrm{PhD}^{1,2}$, \\ William C. Goggins, MD ${ }^{1,2}$ \\ (1) Indiana University School of Medicine \\ (2) Transplant Surgery, Department of Surgery, Indiana University School of Medicine
}

\section{Background:}

At the time of kidney transplantation (KT), induction immunosuppression is used to reduce the incidence of early rejection and avoid the use of chronic corticosteroids in maintenance immunosuppression. There is currently no standard of care for induction immunosuppression in the pediatric recipient, instead it is based on institutional preference. In this study, we compare our current induction immunosuppression, rabbit anti-thymocyte globulin (rATG), to our previous induction immunosuppression, Daclizumab in patients under the age of 11.

\section{Methods:}

From $07 / 2004$ to $08 / 2019,79$ patients under the age of 11 have received a KT. 7 patients were excluded from analysis due to Basiliximab induction $(n=3)$, graft loss within 10 days $(n=3)$ and patient death $(n=1) .72$ patients were analyzed, of which 39 patients $(54 \%)$ with rATG induction were compared to 33 patients $(46 \%)$ with daclizumab induction. All patients were maintained on steroid-free immunosuppression regimen after transplant. More than 20 variables were followed, along with rejection, graft failure, and any prevalence of post-transplant lymphoproliferative disorder (PTLD) was recorded (Figure 1).

\section{Results:}

Patients demographics were similar in both groups. Graft survival was good and statistically similar up to 5 years. In both groups, serum creatinine levels were similar up to 1 year follow up. Although CMV infection was similar in both groups, BK viremia and BK virus in the urine were more frequent in rATG group. Post-transplant lymphoproliferative disorder was significantly higher in the Daclizumab group $(p=0.022)$, but less acute rejection was observed in the Daclizumab group (Figure 1).

\section{Potential Impact:}

Our study suggests that rATG is a safe and effective induction agent in pediatric kidney transplant recipients under the age of 11. Recipients have excellent patient and graft survival. It is associated with strong kidney function and low PTLD. Screening for BK virus in the urine is essential with rATG induction. 
Table 1:

\begin{tabular}{|c|c|c|c|}
\hline Induction Agent & Daclizumab & rATG & $p$ value \\
\hline \multicolumn{4}{|l|}{ Demographics } \\
\hline Number & 33 & 39 & N.S. \\
\hline Sex & $15 \mathrm{M}, 18 \mathrm{~F}$ & $27 \mathrm{M}, 12 \mathrm{~F}$ & 0.042 \\
\hline Age (years) & $5.5 \pm 2.7$ & $6.1 \pm 2.7$ & N.S. \\
\hline Height (m) & $1.02 \pm 0.23$ & $1.06 \pm .21$ & N.S. \\
\hline Weight (kg) & $18.75 \pm 9.93$ & $19.08 \pm 6.42$ & N.S. \\
\hline \multicolumn{4}{|l|}{ Outcomes } \\
\hline Cr 1 month (mg/dL) & $0.56 \pm .31$ & $0.45 \pm .17$ & 0.056 \\
\hline Cr 6 months (mg/dL) & $0.54 \pm .22$ & $0.52 \pm .18$ & N.S. \\
\hline Cr 1 year $(\mathrm{mg} / \mathrm{dL})$ & $0.63 \pm .27$ & $0.59 \pm .17$ & N.S. \\
\hline $\begin{array}{l}\text { eGFR } 1 \text { month } \\
\left(\mathrm{ml} / \mathrm{min} / 1.73 \mathrm{~m}^{2}\right)\end{array}$ & $84.81 \pm 27.95$ & $107.08 \pm 30.09$ & 0.0019 \\
\hline $\begin{array}{l}\text { eGFR } 6 \text { months } \\
\left(\mathrm{ml} / \mathrm{min} / 1.73 \mathrm{~m}^{2}\right)\end{array}$ & $85.04 \pm 27.60$ & $92.48 \pm 28.07$ & N.S. \\
\hline $\begin{array}{l}\text { eGFR } 1 \text { year } \\
\left(\mathrm{ml} / \mathrm{min} / 1.73 \mathrm{~m}^{2}\right)\end{array}$ & $74.31 \pm 26.8$ & $79.3 \pm 22.01$ & N.S. \\
\hline Rejection 6 months & $1(3.03 \%)$ & $8(20.51 \%)$ & 0.0188 \\
\hline Rejection 1 year & $2(6.06 \%)$ & $8(20.51 \%)$ & 0.0682 \\
\hline Graft Survival 1 year & $100 \%(33 / 33)$ & $100 \%(39 / 39)$ & N.S. \\
\hline Graft Survival 3 years & $96.97 \%(32 / 33)$ & $100 \%(25 / 25)$ & N.S. \\
\hline Graft Survival 5 years & $96.88(31 / 32)$ & $100 \%(22 / 22)$ & N.S. \\
\hline Cases of PTLD & $5(18.18 \%)$ & $0(0 \%)$ & 0.022 \\
\hline Chronic steroid use & $2(6.06 \%)$ & $2(5.13 \%)$ & N.S. \\
\hline BK Urine only 1 year & $0 \%(0 / 33)^{*}$ & $10.26 \%(4 / 39)$ & 0.0439 \\
\hline BK Viremia 1 year & $3.03 \%(1 / 33)^{*}$ & $17.95 \%(7 / 39)$ & 0.0356 \\
\hline CMV Viremia 1 year & $0 \%(0 / 33)$ & $5.13 \%(2 / 39)$ & N.S. \\
\hline
\end{tabular}

N.S. = Not statistically significant.

${ }^{*} \mathrm{BK}$ screening was not routine during time of daclizumab induction 